\title{
Simulation of XSTS imaging of self assembled quantum dot electronic states
}

\author{
Mervyn Roy*, P A Maksym*, D M Bruls ${ }^{\dagger}$, P Offermans ${ }^{\dagger}$ and P M \\ Koenraad $^{\dagger}$ \\ ${ }^{*}$ Department of Physics and Astronomy, University of Leicester, Leicester, LE1 7RH, UK. \\ ${ }^{\dagger}$ COBRA Inter-University Research Institute, Eindhoven University of Technology, P.O. Box 513, \\ 5600 MB Eindhoven, The Netherlands
}

\begin{abstract}
Recently, STM measurements of cleaved, self assembled quantum dots (SAQDs) have provided important information on the morphology and composition of these buried semiconductor islands. It is also now becoming possible to use STM techniques to image the electronic charge density within the SAQDs. At low bias voltages, the tunnelling current measured during crosssectional scanning tunnelling spectroscopy (XSTS) experiments contains direct information on the $0 \mathrm{D}$ bound electronic states of the cleaved quantum dots.

In this paper we present a numerical simulation of an XSTS experiment. The calculated tunnelling currents between an STM tip and the bound states inside a physically realistic model of a cleaved SAQD are compared to experimental results and qualitative agreement is found.

The calculation of the tunnelling current is split into two stages. First the bound electron states of the cleaved quantum dot are calculated by exact diagonalisation of the Hamiltonian in a simple harmonic oscillator basis set. The calculation is performed within the single-band effective mass approximation including the position dependence of the effective mass and, most crucially, the effect of the deformations of the cleaved dot structure and the strain field within the system. The strains and deformations of the heterostructure are found with a continuum, finite element model. The calculation method is completely general, however, in this paper we apply it to the InGaAs dot structure reported by Bruls et al [1]. Second, the Tersoff-Hamann approximation [2] is used to calculate the tunnelling probability between the bound electronic states and the STM tip at different tip positions and bias voltages. The calculated STM signal is compared to experimental data and reasonable agreement is obtained. The method may be used to obtain additional physical information about the buried SAQDs, for example, details on the lateral variations in the composition.
\end{abstract}

\section{INTRODUCTION}

Self-assembled quantum dots (SAQDs) are fully quantised atom-like systems in the solid state. Over the past decade there has been a large amount of interest in these structures, mainly due to the potential for applications, for example in quantum information processing and optoelectronic devices. The operating properties of such dot-based devices depend on the form of the bound states of each individual SAQD which, in turn, depend on the physical dot structure. Recently, STM images of cleaved quantum dots have been used to provide detailed physical information on the shape, size and composition profile of the dots, while cross-sectional scanning tunnelling spectroscopy (XSTS) may be used to image the bound states within individual SAQDs.

We simulate an XSTS experiment on a cleaved quantum dot and compare the results to real experimental data. The purpose of this simulation is twofold. First, the experimental 


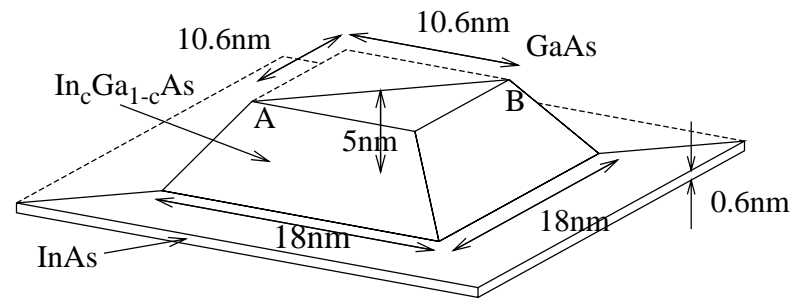

FIGURE 1. Schematic of the dot model investigated by Bruls et al.

data can be used to directly test methods for the calculation of the bound states. Second, with a combination of the experimental and simulated results it may be possible to deduce additional information on the physical structure of the dot from that obtained with XSTM and other techniques.

The paper is split into two sections. We begin by reporting the first calculation of the bound electronic states inside a cleaved quantum dot, including the full details of the dot deformation and the strain field inside the system. The bound wavefunctions are calculated by exact diagonalisation of the single-band, position dependent effective mass Hamiltonian in a harmonic oscillator (HO) basis set. In the second section we use the Tersoff-Hamann approximation [2] to relate the calculated wavefunctions to the tunnelling currents measured in XSTS experiments. The calculated XSTS images are then compared to experimental data.

\section{THE BOUND STATES IN A DEFORMED CLEAVED DOT}

\section{The dot model}

To compare directly with the XSTS experiment we use a dot model with dimensions and composition identical to those measured by Bruls et al [1] with XSTM. The calculational methods described in this paper are, however, completely general and may be used to calculate the XSTS signal from cleaved SAQDs of arbitrary shape, size and composition profile.

The uncleaved Bruls dot (shown by the solid and dashed lines in figure 1) is a square based, truncated pyramidal $\mathrm{In}_{c} \mathrm{Ga}_{1-c}$ As dot with an Indium fraction varying linearly from $c=0.6$ at the base of the dot to $c=1$ at the top of the dot. The SAQD rests on a $0.6 \mathrm{~nm}$ InAs wetting layer and is surrounded by a GaAs matrix. The GaAs cap and substrate have height $30 \mathrm{~nm}$. The origin of our coordinate system is chosen to be at the centre of the uncleaved $\operatorname{In}_{c} \mathrm{Ga}_{1-c}$ As dot. The $z$ axis is taken to be in the growth direction and the $y$ direction is perpendicular to the dot/vacuum interface.

The SAQD is cleaved along the line $\mathrm{AB}$ in figure 1 exposing a cross-section through the dot. After cleaving, the remaining part of the dot (shown by the solid line in figure 1) relaxes, and deforms outward into the vacuum. We model this deformation and calculate the strain field throughout the deformed dot within the continuum elasticity approximation. This is done with a commercially available, finite element package, 

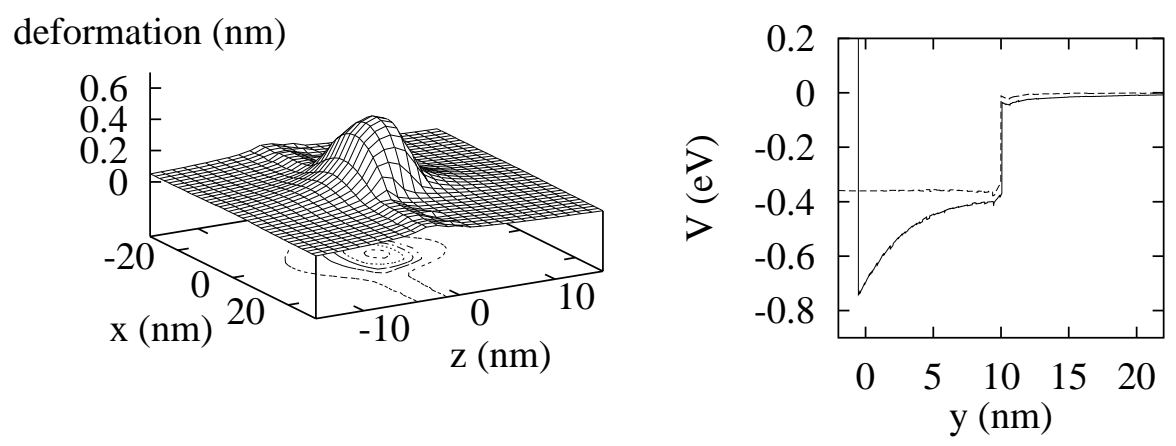

FIGURE 2. Left: calculated deformation of the dot after cleaving. Right: Strained electron confinement potential in the cleaved dot (solid line) compared to the confinement potential in an uncleaved dot (dashed line). Energies are given relative to the GaAs conduction band edge.

Abaqus, which has already been shown to provide an accurate representation of the strain within a cleaved dot [1]. The strain field affects the band gaps in the dot and substrate material and hence alters the electron confinement potential, $V(\mathbf{r})=V_{o}(\mathbf{r})+$ $V_{c}(\mathbf{r})$. The conduction band offset, $V_{o}$, is taken to be $0.797 \mathrm{eV}$ between InAs and GaAs, while the potential step at the dot vacuum interface is set from the electron affinity of GaAs at $4.07 \mathrm{eV}$ above the GaAs conduction band edge. In the $\mathrm{In}_{c} \mathrm{Ga}_{1-c} \mathrm{As}$ material $V_{o}=-1.178 c+0.381 c^{2}$ [3]. The relation between the strain contribution to the confinement potential, $V_{c}(\mathbf{r})$, and the strain is well documented in the literature [5].

Figure 2 shows the calculated deformation of the dot/substrate system after cleaving. The originally highly strained dot and wetting layer regions relax outward into the vacuum in order to minimise the strain energy. The right hand figure shows the calculated $V(\mathbf{r})$ relative to the GaAs conduction band edge. This is much deeper in the cleaved dot (solid line) at the dot/vacuum interface because the material that has deformed outward is less strained than that in the centre of the uncleaved dot (dotted line).

The position dependent effective masses in the semiconductor material are also affected by the strain field. We take the bulk effective masses in GaAs, InAs and the vacuum to be $0.067 m_{o}, 0.023 m_{o}$ and $m_{o}$ respectively and calculate the effective mass in the $\mathrm{In}_{c} \mathrm{Ga}_{1-c}$ As material by linear interpolation. The strained effective masses are then calculated with first order perturbation theory [5]. Finally, for this particular cleaved dot with an exposed (110) surface, we assume flat bands at the dot/vacuum interface, as there are no (110) surface states within the bulk band gap [7].

\section{Calculation method}

To calculate the bound energy levels and the electron wavefunctions we use the singleband effective mass approximation to the Schrödinger equation,

$$
\left[\frac{-{ }^{-h}}{2} \nabla M^{-1} \nabla+V(\mathbf{r})\right] \Psi=E \Psi
$$


where $M$ is the effective mass tensor. We have not yet included the effect of the piezoelectric potential as we expect its effect on the shape of the bound states inside the cleaved dot to be small [4].

Given the position dependent confinement potentials and effective masses we solve equation (1) by expanding the exact wavefunction, $\Psi^{d o t}$, as a sum of $\mathrm{HO}$ functions and then diagonalising the resultant Hamiltonian matrix. In cylindrical polar co-ordinates,

$$
\Psi^{d o t}(\mathbf{r})=\sum_{i} a_{i} \psi_{i}=\sum_{l_{i}=-l_{\max }}^{l_{\max }} \sum_{m_{i}=0}^{m_{\max }} \sum_{n_{i}=0}^{n_{\max }} a_{l_{i} m_{i} n_{i}} \frac{\exp \left(i l_{i} \phi\right)}{\sqrt{2 \pi}} Z_{m_{i}}(z) R_{n_{i} l_{i}}(r),
$$

where the $a_{l_{i} m_{i} n_{i}}$ are expansion coefficients, and we have used $N_{b s}=\left(2 l_{\max }+1\right)\left(m_{\max }+\right.$ 1) $\left(n_{\max }+1\right)$ basis functions to approximate the full single particle wavefunction inside the dot. The individual basis functions in equation (2) are given by,

$$
\begin{aligned}
Z_{m_{i}}(z) & =\left(\frac{\lambda_{z}}{2_{i}^{m} m_{i} ! \sqrt{\pi}}\right)^{\frac{1}{2}} H_{m_{i}}\left[\left(z-z_{o}\right) \lambda_{z}\right] e^{-\left(z-z_{o}\right)^{2} \lambda_{z}^{2} / 2} \\
R_{n_{i} l_{i}}(r) & =\left(\frac{n_{i} ! \lambda_{r}^{2}}{(2 \sqrt{2})\left|l_{i}\right|\left(n_{i}+\left|l_{i}\right|\right) !}\right)^{\frac{1}{2}} e^{-\frac{1}{4} r^{2} \lambda_{r}^{2}}\left(r \lambda_{r}\right)^{\left|l_{i}\right|} L_{n_{i}}^{\left|l_{i}\right|}\left[\frac{1}{2} r^{2} \lambda_{r}^{2}\right],
\end{aligned}
$$

where $H_{m_{i}}(z)$ and $L_{n_{i}}^{\left|l_{i}\right|}(r)$ are Hermite and Laguerre polynomials and $\lambda_{z}$ and $\lambda_{r}$ are the reciprocals of the respective length scales of the HO functions in the parallel and perpendicular directions. The length scales and the offset parameter, $z_{o}$, can be chosen to optimise the rate of convergence of the HO calculation. We choose values of $\lambda_{r}$, $\lambda_{z}$ and $z_{o}$ to give the ground harmonic oscillator basis function a similar spatial extent to the actual localised state within the dot. For the particular cleaved dot investigated, the values used are: $\omega_{o}=0.64 \mathrm{eV}, \omega_{z}=0.54 \mathrm{eV}, z_{o}=0.08 \mathrm{~nm}$. We can also alter the position of the dot/vacuum interface with respect to the origin of the HO functions. With the values of the parameters chosen, the best convergence is obtained by off-setting the interface by $3 \mathrm{~nm}$ in the $y$ direction. This places the maximum in the HO ground state at the approximate position of the maximum in the actual ground state of the dot.

To diagonalise the Hamiltonian and solve for the exact single particle states we must calculate the matrix elements of the Hamiltonian operator (from eqn. (1)) between individual HO states,

$$
H_{j i}=\int\left[\frac{-h}{2 m_{x y}^{*}} \frac{d \psi_{j}^{*}}{d r} \frac{d \psi_{i}}{d r}+\frac{-h}{2 m_{z}^{*}} \frac{d \psi_{j}^{*}}{d z} \frac{d \psi_{i}}{d z}+\psi_{j}^{*} \psi_{i}\left(\frac{-h l_{i} l_{j}}{2 m_{x y}^{*} r^{2}}+V\right)\right] r d r d z d \phi .
$$

Where we have integrated by parts to avoid terms containing the derivatives of the components of the effective mass tensor, which in this particular model must be evaluated numerically. Further details on the calculation method used are reported elsewhere [6].

Once the individual matrix elements have been calculated we diagonalise the Hamiltonian with the LAPACK library standard linear algebra routines. We optimise the computer time for the calculation by using the reflection symmetry of the cleaved dot to block diagonalise the Hamiltonian into 2 blocks. Each block may then be diagonalised separately reducing the total time needed for the diagonalisation by a factor of 4 . 

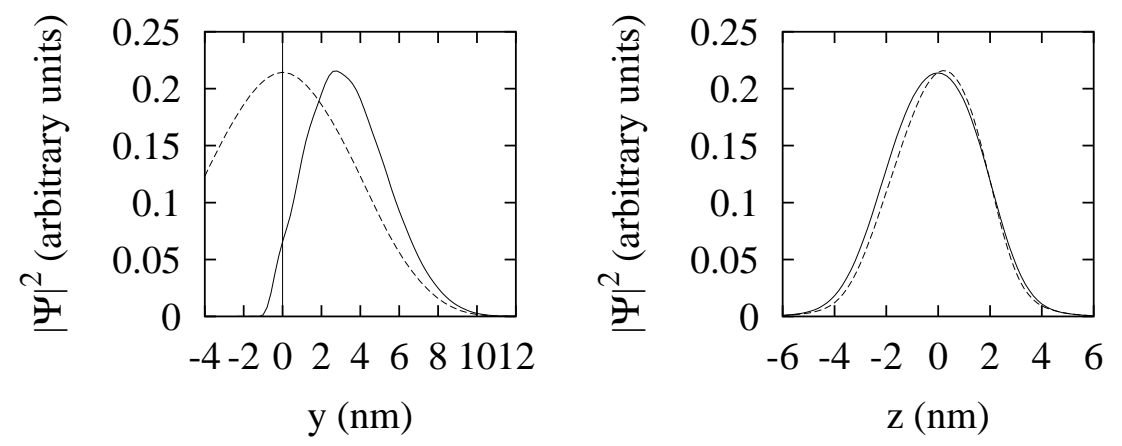

FIGURE 3. Comparison between the ground state charge densities in the cleaved dot (solid line) and original dot (dashed line). Left: variation in the charge density with $y$ at $x=0$ and $z=0$. Right: variation in the charge density with $z$ at $x=0$ and $y=2.8 \mathrm{~nm}$.

\section{Results}

With $21168 \mathrm{HO}$ functions in the expansion of the cleaved dot wavefunctions, we find 4 bound states within the dot at $-0.272 \mathrm{eV},-0.175 \mathrm{eV},-0.102 \mathrm{eV}$ and $-0.072 \mathrm{eV}$. These energies are converged roughly to within $0.5 \mathrm{meV}$ of the exact bound state energies. To obtain results within $1 \%$ of the true energies we still need to include approximately 10000 states in the expansion. This compares unfavourably with a calculation of the states of the original uncleaved dot in which the convergence of the calculation with a HO basis set is rapid: The ground state energy in the uncleaved dot $(-0.225 \mathrm{eV})$ can be converged to within $1 \%$ with only $24 \mathrm{HO}$ functions [6]. This indicates that $\mathrm{HO}$ functions are not the optimum basis for investigating this particular system.

The unintuitive result that the ground state is more tightly bound in the cleaved dot is explained by the deepening of the potential due to the deformation of the dot into the vacuum (see figure 2). The shape of $V(\mathbf{r})$ also explains the poor convergence. It is energetically favourable for the maximum in the charge density to be close to the dot/vacuum interface where the potential is deepest, however the energies of the bound states are sensitive to the tails of the wavefunctions extending into the vacuum. This behaviour can be seen by examining the charge density (figure 3). The maximum in the ground state charge density is at $x=0$ and $z=0$ at the centre of the dot, but at $y=2.8$ $\mathrm{nm}$. This is closer to the interface than was expected before a detailed calculation of the confinement potential in the deformed cleaved SAQD (see figure 2). The left hand plot of figure 3 shows this behaviour: The charge density is appreciable at the edge of the dot but then decays abruptly into the vacuum region. In comparison, the charge density in the uncleaved dot shows a much smoother variation with $y$. From the right hand plot we can see that the charge density in the cleaved dot is slightly more symmetric about $z=0$, than in the original dot. This is because the deformation of the dot tends to even out the variations in strain as a function of $z$.

Figure 4 shows the variation of the bound state charge densities in the $x z$ plane. The excited states all have nodes in the $x$ direction. States 2 and 3 also have nodes in the $y$ direction. The wavefunctions inside the cleaved SAQD are very different to those in the original dot. The overall variation in the charge density, especially in the $x z$ plane is not 

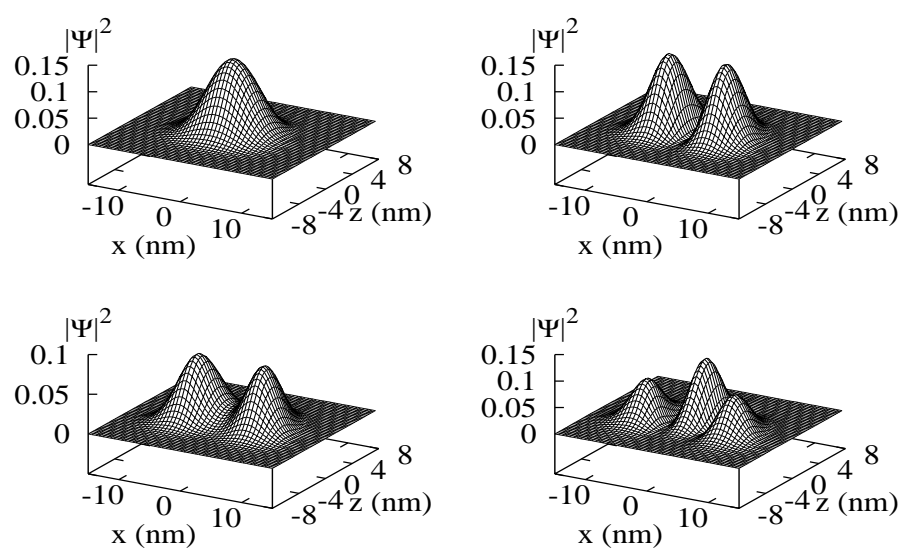

FIGURE 4. Bound state charge densities in the $x z$ plane, plotted at $y=1 \mathrm{~nm}$ from the interface. Ground state (top left). Excited states: 1st (top right), 2nd (bottom left), 3rd (bottom right).

overly sensitive to the number of basis states included in the calculation.

\section{THE XSTS SIGNAL}

\section{Calculation method}

In the XSTS experiment the STM tip is held at a fixed position above the cleaved dot while the variation of tunnelling current with tip bias, $V_{B}$, is measured. At low $V_{B}$ the electrons tunnel from the tip into the OD bound states of the dot. By scanning the STM tip over the exposed surface of the cleaved dot the spatial variation in tunnelling current can be measured, this is related to the spatial variation of the bound states inside the dot. The measured current, $I$, is directly proportional to the tunnelling probability between the tip and dot states. In the Tersoff-Hamann approximation [2] we approximate the tip wavefunction by a decaying spherical wave, then,

$$
I \sim \sum_{n}\left|\int \Psi_{n}^{d o t} \frac{\exp \left(-k_{t i p}\left|\mathbf{r}-\mathbf{r}_{t i p}\right|\right)}{\left|\mathbf{r}-\mathbf{r}_{t i p}\right|} d \mathbf{r}\right|^{2} f_{t i p}\left(E_{f}+E_{n}\right) g_{t i p}\left(E_{f}+E_{n}\right) .
$$

Where $\Psi_{n}^{d o t}$ is the $n$th excited state of the cleaved dot, of energy $E_{n}$. The wavevector of the decaying tip wavefunction is $k_{t i p}=\left[2 m_{o}\left(V_{v a c}+E_{n}\right)\right]^{1 / 2} /{ }^{-} h, f_{i p}$ is the Fermi occupation function of the STM tip, $g_{\text {tip }}$ is the tip density of states and $V_{v a c}$ is the height of the potential step at the dot/vacuum interface. To obtain equation (5) we have assumed the dot states are unoccupied and used the fact the 0D bound states have a delta function density of states. The actual and simulated experiments were performed at room temperature, which we assume to be $290 \mathrm{~K}$. Altering the tip bias voltage experimentally is equivalent to altering the position of the tip Fermi level, $E_{f}$ in this model. $E_{f}$ is measured relative to the ground state of the dot. At low $V_{B}$, or alternatively, low $E_{f}$, the tunnelling is directly into the dot ground state. As $E_{f}$ is increased the total observed current picks up contributions in turn from tunnelling into each of the excited states of the dot. 
In this approximation, the tip wavefunction decays rapidly away from the position of the STM tip. This makes the calculated tunnelling current sensitive to the tails of the bound states extending into the vacuum. In this area, where the magnitude of the wavefunctions are small, there is a significant amount of noise in the calculated results. To average out some of the noise, we impose a muffin tin radius of $0.5 \mathrm{~nm}$ around the tip in which we assume the tip wavefunction to be constant. Physically this corresponds to the dimensions of the STM tip. In a more accurate model we could solve for the wavefunctions inside this region, possibly as an expansion of spherical Bessel functions.

In this model we ignore the tip induced band bending. Calculations with a 1D model indicate that the band bending over the region of the dot can be approximated by a constant offset in the band edges. This has no effect on the charge densities, but makes the relation between $E_{f}$ and the experimentally applied bias voltage unclear.

\section{Results}

In both the experimental and simulated XSTS scans the STM tip is scanned parallel to the deformed dot surface at a distance of $0.6 \mathrm{~nm}$ from the exposed surface. In this paper we compare calculated results with experimental line scans along $x$ at $z=0$ measured at two different bias voltages. To directly compare the experiment and theory we have scaled each of the calculated tunnelling currents by a constant factor and added a constant background. In general, as $E_{f}$ is increased we see an evolution in the calculated XSTS signals similar to that observed experimentally as $V_{B}$ is raised.

On the left hand side of figure 5 we can see that the experimental and calculated tunnelling currents have similar shape when the tunnelling is primarily into the dot ground state. The experimental FWHM is $9.9 \pm 0.9 \mathrm{~nm}$, compared to a calculated result of $11.1 \mathrm{~nm}$. The broad shoulders on the calculated curve come from the contribution to the signal from tunnelling into the 1st excited state within the dot.

At larger $V_{B}$ (right hand side of figure 5) we also obtain similar results to the experimental scans. The separation between the lobes of the charge density is approximately $11.7 \mathrm{~nm}$ (experimental) and 11.1 (calculated). The separation between the minima in the charge density is approximately $9.3 \mathrm{~nm}$ (experimental) and 7.7 (calculated). The ratio between the heights of the central peak and the lobes is similar in both cases.

In conclusion, we have described a method for interpreting the results of XSTS measurements based on the calculated electron wavefunctions within cleaved SAQDs in which the qualitative agreement between experiment and theory is promising. By including such effects as the tip induced band bending, the position dependence of $k_{t i p}$, and the piezoelectric potential in this model, we may be able to obtain better quantitative agreement with experiment. The experimental scans can then be fitted with different dot models to obtain additional information on the physical structure of the dot. 

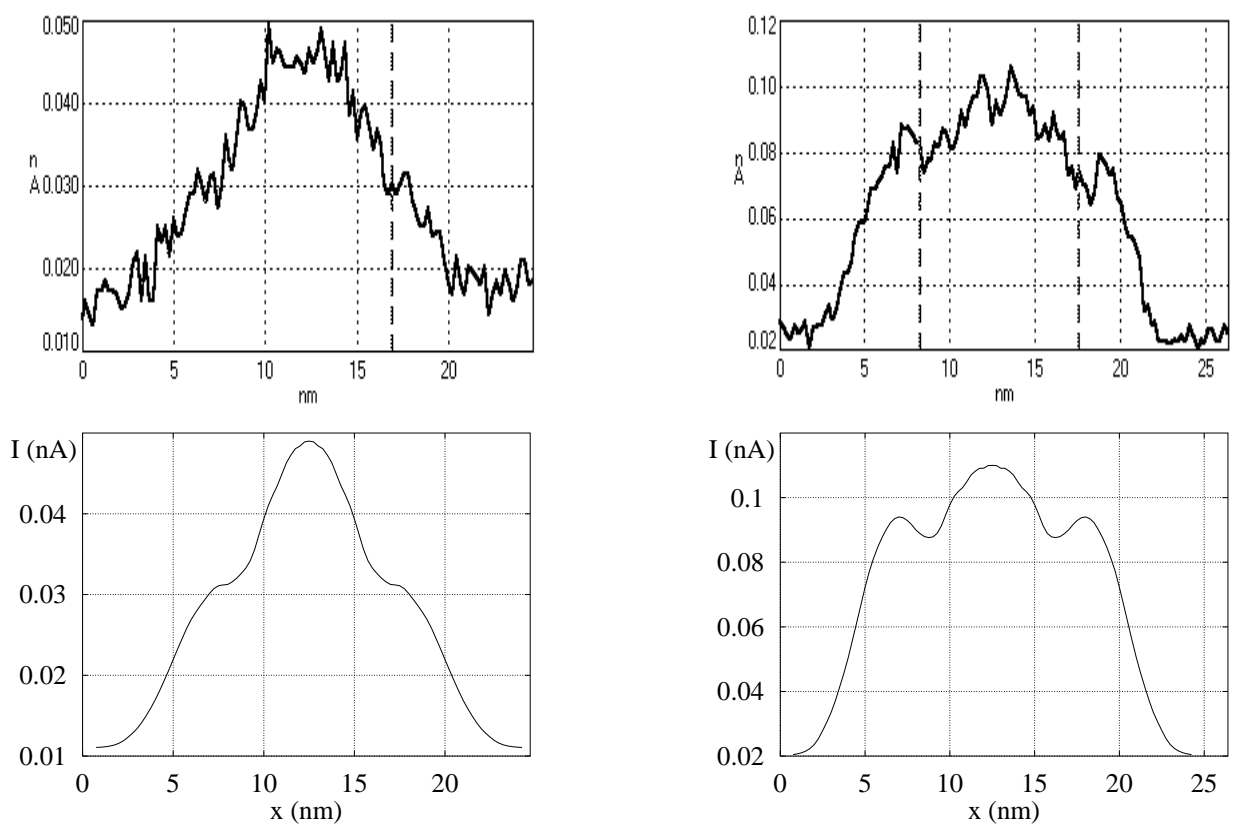

FIGURE 5. Calculated and experimental XSTS signals $0.6 \mathrm{~nm}$ from the interface, along $x$ at $z=0$. Left hand plots: Average of experimental scans between $V_{B}=0.81 \mathrm{~V}$ and $V_{B}=0.9 \mathrm{~V}$ (top), calculated result with $E_{f}=0.02 \mathrm{eV}$ (bottom). Right hand plots: Average of experimental scans between $V_{B}=0.121 \mathrm{~V}$ and $V_{B}=0.13 \mathrm{~V}$ (top), calculated result with $E_{f}=0.08 \mathrm{eV}$ (bottom).

\section{ACKNOWLEDGMENTS}

This work was performed with the University of Leicester Mathematical Modelling Centre supercomputer which was purchased through the EPSRC strategic equipment initiative.

\section{REFERENCES}

1. Bruls, D.M., Vugs, J.W.A.M., Koenraad, P.M., Skolnick, M.S., Hopkinson, M., Long, F., Gill, S.P.A., and Wolter, J.H., Appl. Phys. Lett. 81, 1708 (2002).

2. Tersoff, T., Hamann, D.R., Phys. Rev. B. 31, 805, (1985).

3. Barker, J.A., O' Reilly, E.P., Physica E 4, 231 (1999).

4. Grundmann, M., Stier, O., and Bimberg, D., Phys. Rev. B 52, 11969 (1995).

5. Fonseca, L.R.C., Jimenez, J.L., Leburton, J.P., and Martin, R.M., Phys. Rev. B 57, 4017 (1998).

6. Roy, M. and Maksym, P.A., submitted to Phys. Rev. B.

7. Mönch, W., Semiconductor Surfaces and Interfađes, Springer-Verlag, Berlin, 1995. 